\section{How fast can you get?}

\section{Wolfhard Almers}

NEUROTRANSMITTER release from a presynaptic terminal is one of the fastest things animal cells do - it occurs in a brief burst within the first millisecond of the arrival of an action potential ${ }^{1,2}$. On page 735 of this issue ${ }^{3}$, von Gersdorff and Matthews explore calcium-triggered exocytosis of synaptic vesicles by capacitance measurements. They provide the most direct evidence yet that the calcium sensor for exocytosis is a low-affinity receptor, and demonstrate a hitherto unrecognized, and surprisingly fast, mechanism for endocytosis. They show that, for $100 \mathrm{~ms}$ or more, terminals can continue to release transmitter at rates close to those attained after an action potential $(0.2$ vesicles per millisecond per release zone).

The existence of a low-affinity $\mathrm{Ca}$ sensor had been widely postulated ${ }^{4}$. At the release (or 'active') zones of synaptic terminals, vesicles and voltage-gated $\mathrm{Ca}$ channels co-inhabit microdomains where cytosolic $\left[\mathrm{Ca}^{2+}\right]$ rises in microseconds to near-millimolar levels as individual $\mathrm{Ca}$ channels open, and where $\left[\mathrm{Ca}^{2+}\right]$ falls nearly as quickly when the channels close. For release to stop rapidly after an action potential, the $\mathrm{Ca}$ sensor must let go of its bound Ca quickly. So its low affinity is of critical importance.

The rapid endocytosis revealed by von Gersdorff and Matthews coexists with the more familiar, slower, membrane retrieval probably mediated by clathrincoated pits ${ }^{5,6}$. Similar effects have also been seen after exocytosis of dense core granules ${ }^{7}$. In both cases, the slow mechanism is evident only after massive stimulation, whereas the fast mechanism probably operates throughout. Therefore, for the first time, we may have observed the physiological mechanism of membrane retrieval after $\mathrm{Ca}$-triggered exocytosis in excitable cells.

The new results on the kinetics of release are perhaps the most intriguing, as they have implications for the number of vesicles that a synapse can release rapidly, as well as for the speed of the exocytic machinery. The issues involved are illus- trated in the accompanying box. An 'exocytic time' is defined by asking the following question - once $\mathrm{Ca}$ has bound to the $\mathrm{Ca}$ sensor, how long does the exocytic machinery need on average to accomplish transmitter release? With release occurring in under $1 \mathrm{~ms}$, most of us have concluded that the exocytic time is similarly short. That conclusion follows only if a sizeable portion of the releasable vesicles is, in fact, released by one action potential. And that, in turn, implies that only very few of the vesicles docked at release zones - maybe only one - are readily releasable (see box, model I). For several synapses, this view is supported by analyses of fluctuations in transmitter release during successive stimuli ${ }^{8,9}$. An exocytic time in the millisecond range would generally accommodate only one fast biochemical reaction. Most of the rich synaptic biochemistry discovered over the past few years ${ }^{10-12}$ would have to happen before the action potential arrived at the terminal.

However, if it were not for the statistical findings, fast transmitter release would also be consistent with much longer exocytic times. In model II (see box) the vesicles released are assumed to be drawn from a large pool. Rather than an intrinsi-

\title{
Synaptic transmission: high speed with slow molecules?
}

LARGE synaptic terminals release vesicles from tens to hundreds of release zones, each with dozens of vesicles. As an example, $a$ in the figure shows two release zones, I and II, each with 15 synaptic vesicles docked beneath the plasma membrane, and each before and after an action potential. In II, all 15 vesicles can respond equally to $\mathrm{Ca}$ with exocytosis. In I, only three of the 15 vesicles can readily do so, while the remainder are in a dormant state. Assume that an action potential raises the local $\left[\mathrm{Ca}^{2+}\right]$ next to each vesicle to saturating levels for a period of 1 $\mathrm{ms}(b)$. In response, each terminal releases two vesicles $(c)$.

Although both terminals achieve the same result, terminal I requires a faster exocytic machinery than terminal II, releasing as many vesicles per millisecond while drawing on a fivetimes smaller pool. In I, the pool of releasable vesicles is twothirds exhausted within $1 \mathrm{~ms}$ of the advent of high $\left[\mathrm{Ca}^{2+}\right]$, while in II exhaustion is only 10 per cent. To make this more precise, assume that a single reaction is rate-limiting in exocytosis, and that $\left[\mathrm{Ca}^{2+}\right]$ remains high indefinitely (dashed

b.
I
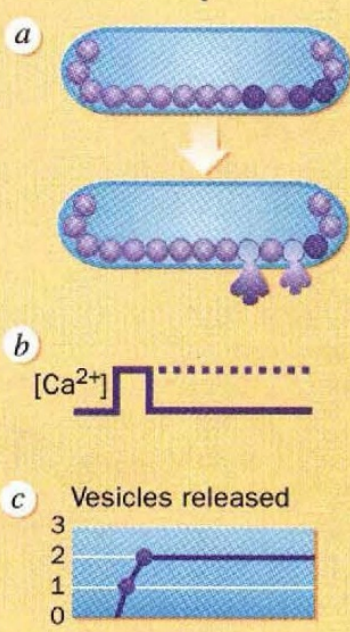

d Releasable vesicles remaining

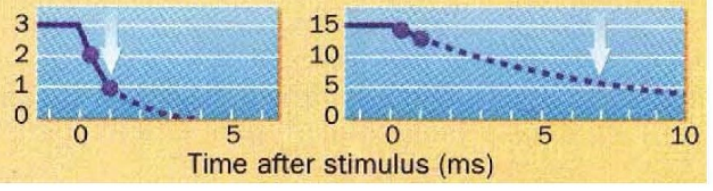

mean time for an exocytosiscompetent vesicle to perform exocytosis at saturating $\left[\mathrm{Ca}^{2+}\right]$. It is $7 \mathrm{~ms}$ in 11 and $0.9 \mathrm{~ms}$ in I, hence the exocytic machinery is seven times faster in I. To achieve speed, II relies on a rapid rise and fall of $\left[\mathrm{Ca}^{2+}\right]$, on a large number of vesicles and on statistics to ensure that a lucky few have enough thermal energy to complete exocytosis ahead of schedule.

The validity of these two mod. els can be tested by observing fluctuations in transmitter release from stimulus to stimulus and measuring their variance. Model II predicts that the variance equals the mean number of vesicles released, model I that it is less. Experiments favour model $I$. The figure also suggests that the exocytic time can be measured directly by forcing a maintained step increase in cytosolic $\left[\mathrm{Ca}^{2+}\right]$, by observing the exhaustion of rapidly releasable in $b)$. In this case (d) exocytosis exhausts the pool of releasable vesicles along an exponential function (dashed), with $1 / e$ of the vesicles remaining after one time constant, $\tau$ (arrows in $d$ ). $\tau$ also equals the 'exocytic time', defined here as the vesicles during exocytosis and by measuring the time constant of exhaustion. In neuroendocrine cells secreting dense core granules, such measurements indicate an exocytic time of $40 \mathrm{~ms}$ at room temperature ${ }^{13}$. 